\title{
Indoor Off-Body Wireless Communication Using Static Zero-Elevation Beamforming on Front and Back Textile Antenna Arrays
}

\author{
Patrick Van Torre* ${ }^{* \dagger}$ Luigi Vallozzi*, Hendrik Rogier*, Jo Verhaevert ${ }^{\dagger}$ \\ *Department of Information Technology, Ghent University, Sint-Pietersnieuwstraat 41, B-9000 Ghent, Belgium \\ ${ }^{\dagger}$ Department INWE, University College Ghent, Valentin Vaerwyckweg 1, B-9000 Ghent, Belgium \\ patrick.vantorre@ugent.be, luigi.vallozzi@ugent.be, hendrik.rogier@intec.ugent.be, jo.verhaevert@hogent.be
}

\section{INTRODUCTION}

Off-body wireless communication systems may be life saving for rescue workers operating in an indoor environment. A system including antennas and sensors integrated into their garment allows communication of vital measurement data to a base station in order to improve the safety and security of the rescue workers and the coordination of their intervention in general. The indoor propagation environment introduces space and time varying channel behavior, including fading, shadowing and non-constant path loss. Specifically for offbody wireless communication links, the movements of the person wearing the communication system will create altering shadowing by the human body, as well as antenna reorientation.

As the reliability of the link is of paramount importance for the monitoring of workers in an emergency situation, systems consisting of multiple textile antennas distributed over the body of the wearer are used to improve the robustness of the link. Such an enhancement is possible by employing transmit and/or receive diversity, using space-time coding and maximal ratio combining, respectively.

Transmit beamforming techniques are able to produce a higher average signal-to-noise ratio at the receiver by concentrating the available transmission power towards the receiver. Dynamic beamforming techniques, using channel estimates to determine the optimal set of transmitted signals, theoretically offer the best performance. However, their implementation for off-body communication with a moving person requires high-rate channel feedback, increasing the complexity, cost and power-consumption of battery-operated systems beyond acceptable limits.

For this paper we present measurements for a static off-body beamforming system. Measurements have been described in literature for outdoor-indoor scenarios [1] but not for indoor off-body beamforming. The off-body system includes two vertically oriented uniform linear arrays consisting of four textile patch antennas [2] at the front and back of the human body, drastically reducing the body's shadowing effect.

Previous measurements with only a single front antenna array are described in [3]. The new measurement campaign here documents the performance of a system deploying both front and back textile antenna arrays on the human body in similar conditions. Both arrays will be driven with the same signals for equal patch numbers, resulting in a more omnidirectional radiation pattern in the azimuth plane. This configuration significantly reduces the shadowing by the human body.

\section{MEASUREMEnT SETUP}

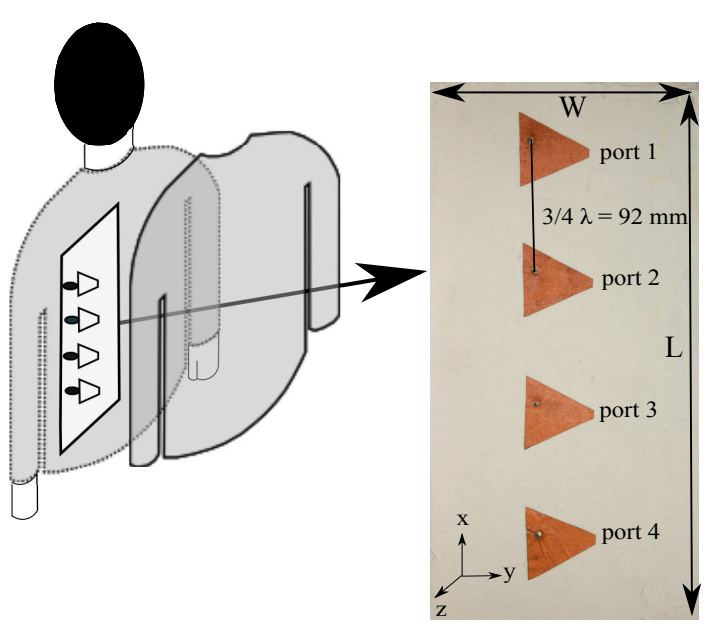

Fig. 1. Array of four textile patch antennas integrated into the firefighters' garment.

Figure 1 displays the textile antenna array and its position on the human body. A tip-truncated equilateral triangular topology is used for each patch, which leads to low mutual coupling while still providing sufficient gain in the broadside direction, i.e. away from the rescue worker. The antenna patches are driven in phase to produce a zero-elevation beam, concentrating the power towards a receiver situated on the same floor. The performance of this beamforming array is compared to a space-time coding system using the same off-body antenna configuration and the corresponding signalto-noise and bit error characteristics are presented. In the measurement campaign with one textile antenna array [3], the performance is highly influenced by shadowing effects of the human body. Nevertheless, a higher average received signalto-noise ratio is always obtained by means of beamforming. 
However, receiver diversity is essential, as the resulting reduction in signal variance is required to produce acceptable bit error characteristics in the demanding indoor propagation environment.

The system shown in Fig. 2 realizes fourth-order receiver diversity at the access point side using a horizontal array of four vertical dipoles. The dipole antennas are connected to the measurement testbed which down converts and synchronously samples the signals.

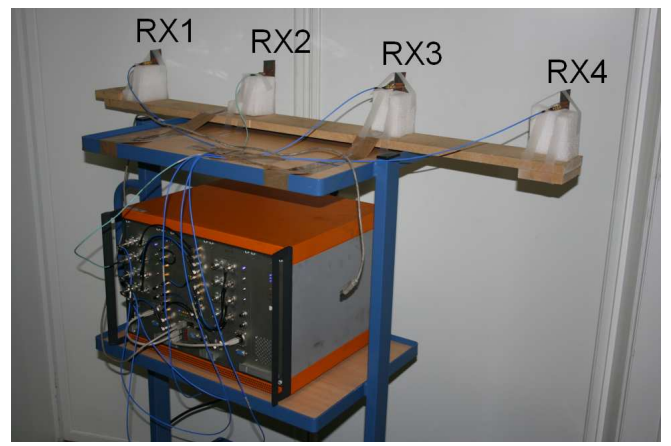

Fig. 2. Measurement equipment at the receiving side, including horizontal four-dipole array.

A floor plan of the measurement environment is displayed in Fig. 3. The Line-of-Sight (LoS) path is situated between the labels A and B, whereas the Non Line-of-Sight (NLoS) path is present between $\mathrm{A}$ and $\mathrm{C}$. Along the LoS path the rescue worker walks towards or away from the transmitter, resulting in a constantly changing path loss. The NLoS path, marked "sideways", is oriented approximately perpendicular to the line between the transmitter and receiver. A nearly constant path loss results. The environment contains office equipment such as metal PC cases and metal closets. The thickest walls in the floor plan are solid brick, building-supporting walls. The NLOS path is blocked from the receiver by two such walls. Other walls are also constructed in brick except for the thinnest walls, which are plasterboard.

\section{AnAlysis of THE RECEIVED $E_{b} / N_{0}$}

$E_{b} / N_{0}$ refers to the ratio of the received energy per bit to the one-sided spectral noise density, a value that is equal for space-time coding and beamforming for our experiments, under equal channel attenuation conditions. An overview of the average, minimum and maximum $E_{b} / N_{0}$ received for different situations is listed in Table I. Beamforming power gain is the difference between the average $E_{b} / N_{0}$ in $\mathrm{dB}$ for beamforming and space-time coding. Because of the concentration of power in low elevation angles this factor amounts to approximately $3 \mathrm{~dB}$ for all measured cases. In [3], a slightly higher beamforming gain results for the LoS situations with the beam directed towards the receiver because the power is more concentrated in a narrower range of azimuth angles when employing only one antenna array. When, for the dual-array system, the front array is directed towards the receiver, half of the power is radiated at the back array, away from the receiver.

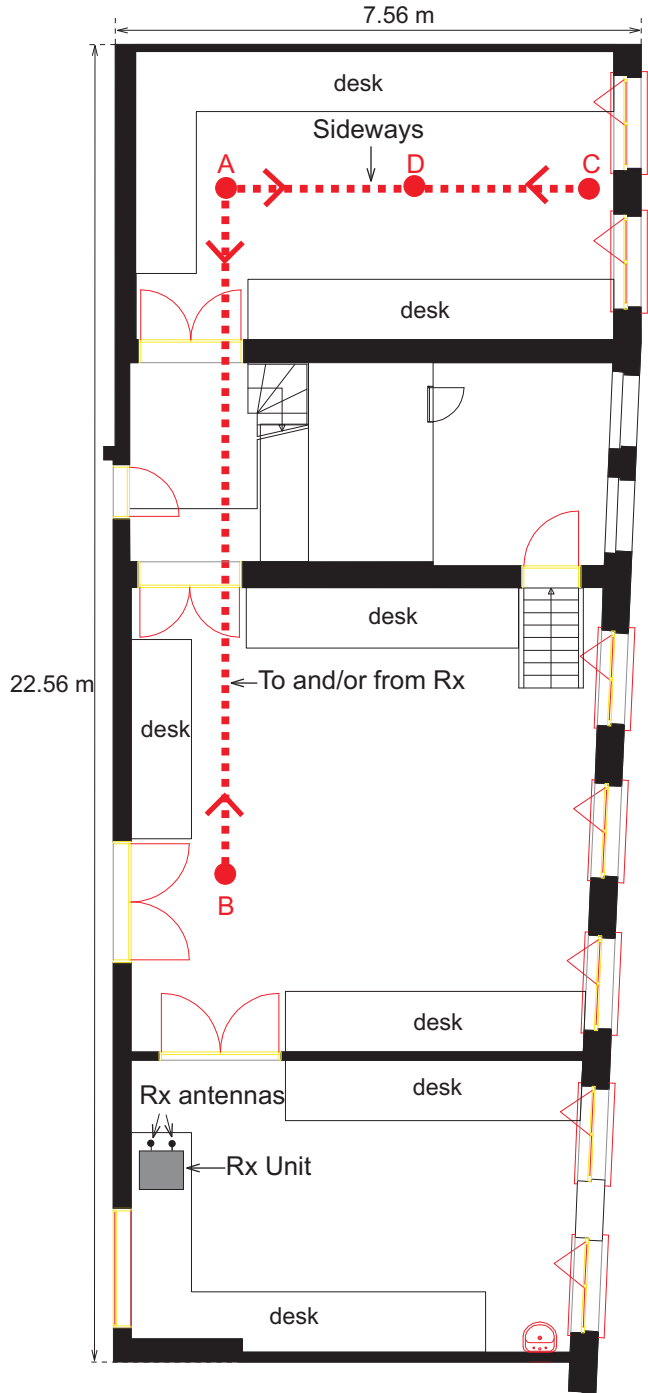

Fig. 3. Floor plan of the measurement environment.

TABLE I

$E_{b} / N_{0}$ FOR STC AND BEAMFORMING; BEAMFORMING POWER GAIN

\begin{tabular}{|c|c|c|c|}
\hline & $\begin{array}{l}\text { STC } \\
{[\mathrm{dB}]}\end{array}$ & $\begin{array}{l}\text { Beamforming } \\
{[\mathrm{dB}]}\end{array}$ & $\begin{array}{l}\text { Beamforming power gain } \\
{[\mathrm{dB}]}\end{array}$ \\
\hline \multicolumn{4}{|c|}{ NLoS, no receive diversity } \\
\hline $\min$ & 34.1 & 19.3 & \multirow{3}{*}{2.9} \\
\hline avg & 44.1 & 47.0 & \\
\hline $\max$ & 50.8 & 55.4 & \\
\hline \multicolumn{4}{|c|}{ NLoS, 4-th order receive diversity } \\
\hline $\min$ & 41.6 & 43.9 & \multirow{3}{*}{3.4} \\
\hline avg & 51.0 & 54.4 & \\
\hline $\max$ & 57.1 & 61.7 & \\
\hline \multicolumn{4}{|c|}{ LoS, no receive diversity } \\
\hline $\min$ & 33.5 & 37.5 & \multirow{3}{*}{2.8} \\
\hline avg & 53.2 & 56.0 & \\
\hline $\max$ & 59.9 & 62.3 & \\
\hline \multicolumn{4}{|c|}{ LoS, 4 -th order receive diversity } \\
\hline $\min$ & 49.7 & 48.5 & \\
\hline avg & 59.4 & 62.3 & 2.9 \\
\hline $\max$ & 65.6 & 68.9 & \\
\hline
\end{tabular}




\section{A. Line-of-Sight}

The $E_{b} / N_{0}$ for subsequent frames received along the LoS path, is displayed in Fig. 4 and 5. Clearly, the shadowing by the human body is countered by the dual antenna array, installed front and back. For the 2-array measurement considered in this paper, the behavior of the $E_{b} / N_{0}$ is dominated by changing path loss and fading. Measurements in [3] show a large drop in signal level when the antenna array is oriented away from the receiver, due to body shadowing combined with the radiation pattern of the single array. In comparison, the $E_{b} / N_{0}$ variance is much smaller for the dual-array system, which will result in better bit error (BER) characteristics.

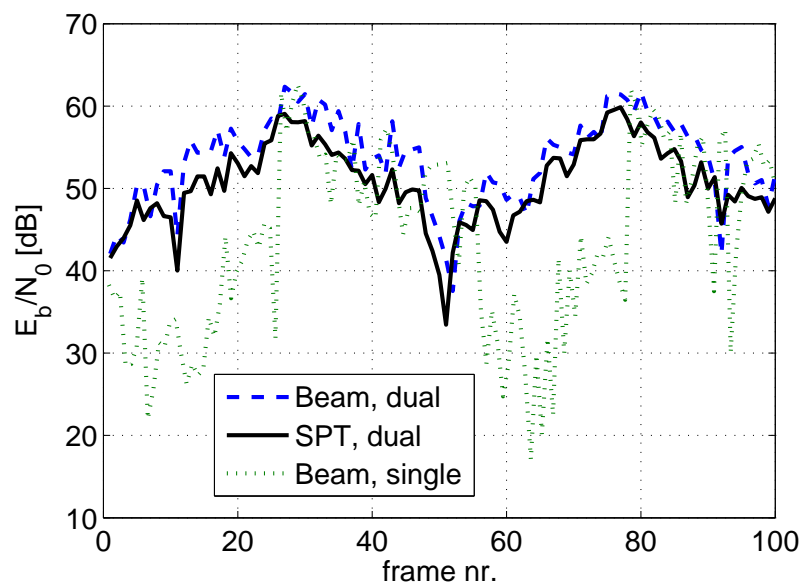

Fig. 4. $E_{b} / N_{0}$ along the Line-of-Sight path, without receiver diversity (RX3) for beamforming and space-time coding.

1) LoS without receiver diversity: Without receiver diversity, beamforming produces an $E_{b} / N_{0}$ that is nearly always higher than for space-time coding. The average $E_{b} / N_{0}$ is $2.8 \mathrm{~dB}$ higher for beamforming than for space-time coding. The minimum $E_{b} / N_{0}$ values for space-time coding and beamforming are $33.5 \mathrm{~dB}$ and $37.5 \mathrm{~dB}$, respectively, for the dualarray system. For the single array configuration [3], those values were $13.1 \mathrm{~dB}$ and $22.3 \mathrm{~dB}$, respectively, when the beam was oriented away from the receiver. Hence, for LoS, the signal minima are much less severe for the dual-array configuration.

2) LoS with 4th order receiver diversity: Although thanks to the array gain the average $E_{b} / N_{0}$ is approximately $6 \mathrm{~dB}$ higher, similar results are obtained for the system with 4-th order receiver diversity, for the beamforming as well as for the space-time coding. Additional diversity gain limits the signal dips, with a minimum $E_{b} / N_{0}$ that is $11.0 \mathrm{~dB}$ and $16.2 \mathrm{~dB}$ higher than without receiver diversity, for beamforming and space-time coding respectively.

\section{B. Non Line-of-Sight}

1) NLoS without receiver diversity: Fig. 6 displays the behavior for NLoS without receiver diversity. For the NLoS environment, beamforming still produces a higher average $E_{b} / N_{0}$ but due to the absence of diversity, serious fading is

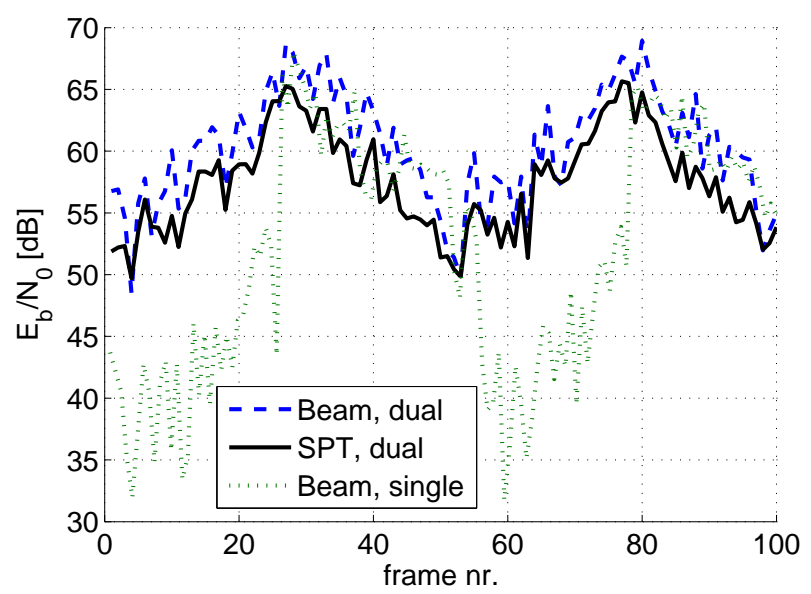

Fig. 5. $E_{b} / N_{0}$ along the Line-of-Sight path, with receiver diversity (MRC) for beamforming and space-time coding.

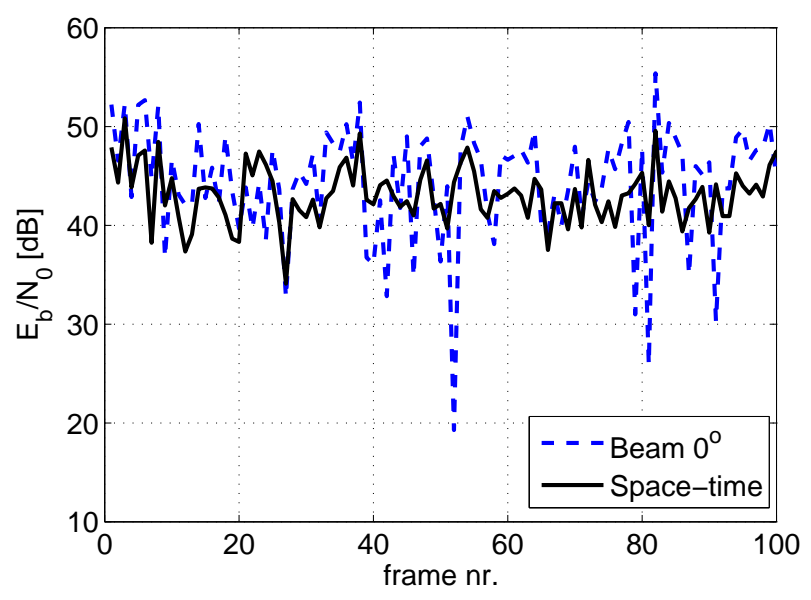

Fig. 6. $E_{b} / N_{0}$ along the Non Line-of-Sight path, without receiver diversity (RX3) for beamforming and space-time coding.

present on the signal. Clearly the space-time code is successful in reducing the fading, indicating that even though the antenna patches in the array are closely spaced, a useful degree of diversity is obtained. Several signal dips occur for the beamforming, with a minimum $E_{b} / N_{0}$ that is $14.8 \mathrm{~dB}$ lower than for the space-time coding (Table I). As further illustrated, these signal dips will significantly deteriorate the bit error characteristics for beamforming.

2) NLoS with 4th order receiver diversity: The NLoS performance with 4 -th receiver diversity is shown in Fig. 7. The average $E_{b} / N_{0}$ is $3.4 \mathrm{~dB}$ higher for the beamforming than for the space-time coding. This value is even higher than for the LoS situation, which indicates that the NLoS signal propagation over larger distances occurs mainly through reflections and scattering at low elevation angles. Comparing to the NLoS situation without receiver diversity, the average $E_{b} / N_{0}$ is $6.9 \mathrm{~dB}$ and $7.4 \mathrm{~dB}$ higher for space-time coding and beamforming, respectively, because of the receiver array and diversity gain combined. 


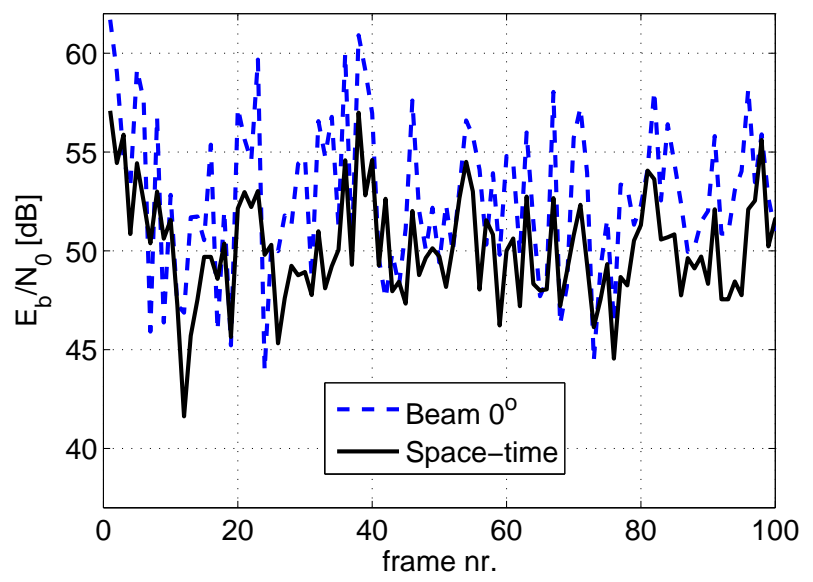

Fig. 7. $E_{b} / N_{0}$ along the Non Line-of-Sight path, with receiver diversity (MRC) for beamforming and space-time coding.

In the presence of receiver diversity, the performance of beamforming is much better because the signal dips are also drastically reduced. Now, the minimum $E_{b} / N_{0}$ occurring for the space-time coding is $2.3 \mathrm{~dB}$ lower than for beamforming. Although the combination of transmit and receiver diversity provides 16-th order diversity for the space-time coding and only 4-th order diversity for the beamforming, the increase in diversity gain offered by the space-time coding is smaller than the power gain produced by beamforming.

\section{BIT ERROR CHARACTERISTICS}

The bit error characteristics display the bit error rate (BER) obtained for beamforming and space-time coding, with and without diversity in LoS as well as NLoS conditions. To obtain a fair comparison of the BER produced by beamforming versus space-time coding, we consider an equal total transmitted energy per information bit $E_{b, t r}$ for both scenarios. Therefore, we introduce the notion of normalized average $E_{b} / N_{0}$, which equals either the average $E_{b} / N_{0}$ at the detector output (in the case of STC), or the average $E_{b} / N_{0}$ at the detector output minus the beamforming power gain from Table I (in the case of beamforming). In this way, displaying the BER curves as a function of the normalized $E_{b} / N_{0}$ includes the power gain associated with coherent beamforming.

\section{A. Line-of-Sight}

Along the LoS path a lower BER is obtained for the beamforming without receiver diversity, because of the beamforming power gain. In case of receiver diversity, the beamforming power gain is also visible but the space-time coding appears to perform better for the lower BERs. Note that the curve for the space-time coding probably bends down excessively for the lower bit error rates, due to the limited number of measurement points. Looking back at Fig. 5 there's only one frame where the $E_{b} / N_{0}$ for beamforming is lower than for space-time coding. Except for this single frame, beamforming always performs better here. However this single frame has a large impact on the lower end of the bit error curve.

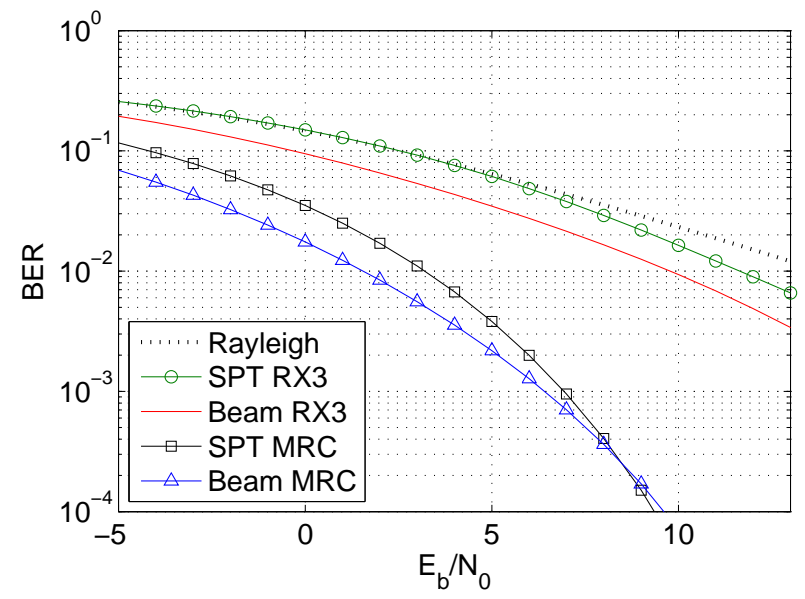

Fig. 8. BER as a function of the normalized average $E_{b} / N_{0}$ per receive antenna, recorded along the LoS path, for transmissions at equal total $E_{b, t r}$.

\section{B. Non Line-of-Sight}

In NLoS conditions without receiver diversity, the spacetime code clearly outperforms the beamforming. The absence of diversity results in a beamforming characteristic approaching the theoretical curve for Rayleigh fading. For the higher $E_{b} / N_{0}$ values, a lower BER is quickly obtained by the spacetime coding, because of the transmit diversity gain exceeding the beamforming power gain.

In case of receiver diversity, the beamforming BER is lower than the BER for space-time coding over the full range displayed. For the beamforming, 4-th order diversity is present whereas the space-time code realizes 16 -th order diversity. The extra diversity gain achieved by space-time coding is minimal for the BER range considered. Possibly the space-time code is better for very low bit error rates, but the number of measured frames is too low to accurately determine this.

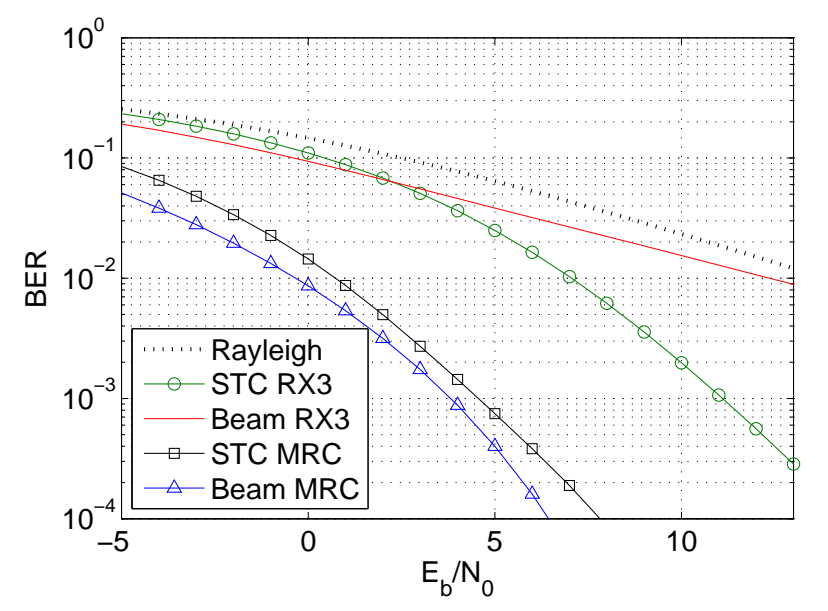

Fig. 9. BER as a function of the normalized average $E_{b} / N_{0}$ per receive antenna, recorded along the NLoS path, for transmissions at equal total $E_{b, t r}$. 


\section{COMPARISON OF SINGLE- AND DUAL-ARRAY SYSTEMS}

An earlier measurement campaign in similar propagation conditions with a single front antenna array is documented in [3]. A comparison of different measurement campaigns should be made carefully because the path traveled by the rescue worker can never be exactly the same. However, some differences between single- and double-array systems are clearly visible. Note that, whereas in [3] the measurements for the LoS path are split up in cases with the beam towards and away from the receiver, this is not the case for the following comparison.

Table II lists the transmit power needed per channel (4 transmitter output channels are used in all cases) to produce an average BER $=10^{-4}$ at the receiver for different antenna and diversity configurations and for the LoS and NLoS measurement scenarios. In the table, MRC refers to 4-th order receiver

TABLE II

TRANSMIT POWER PER CHANNEL FOR A BER OF $10^{-4}$

\begin{tabular}{ccccc}
\hline & \multicolumn{2}{c}{ LoS } & \multicolumn{2}{c}{ NLoS } \\
& $\begin{array}{c}\text { Beam } \\
{[\mathbf{d B m}]}\end{array}$ & $\begin{array}{c}\text { STC } \\
{[\mathbf{d B m}]}\end{array}$ & $\begin{array}{c}\text { Beam } \\
{[\mathbf{d B m}]}\end{array}$ & $\begin{array}{c}\text { STC } \\
{[\mathbf{d B m}]}\end{array}$ \\
\hline \multicolumn{4}{c}{ 1 front array } \\
RX3 & -12.0 & -20.9 & -3.9 & -8.2 \\
MRC & -28.5 & -27.7 & -15.0 & -16.4 \\
& $\mathbf{2}$ arrays, front and back & \\
RX3 & -33.2 & -29.1 & +5.0 & -9.7 \\
MRC & -43.7 & -44.0 & -18.5 & -17.2 \\
\hline
\end{tabular}

diversity, whereas RX3 refers to reception at only antenna 3, situated in the middle of the receiving array in Fig. 2. Systems with $\mathrm{MRC}$ require approximately 10 times less transmit power, compared to reception at RX3. In case of MRC, beamforming and space-time coding require nearly the same transmit power. Note that static beamforming can be realized using only power splitters en is therefore much more low-cost than space-time coding, which requires full transmitter chains for each channel. Without MRC, beamforming is often not efficient, due to the absence of diversity in the link.

The double array system requires significantly less transmit power than the single array configuration for equal signaling and propagation conditions. The associated extra system gain for the MRC cases is highest in LoS conditions, amounting to $15.2 \mathrm{~dB}$ for beamforming and $16.3 \mathrm{~dB}$ for space-time coding. In NLoS conditions these values are $3.5 \mathrm{~dB}$ and $1.2 \mathrm{~dB}$, respectively.

The single array system with the array oriented towards the receiver in LoS conditions, performs better than the dualarray system, with an up to $5 \mathrm{~dB}$ better BER characteristic [3] and comparable average received signals. However, in realistic conditions the beam will be directed randomly as the rescue worker is performing his duties, and a much better average performance for the dual-array system results.

In NLoS conditions with MRC, the required transmit power is comparable for dual- and single-array systems, allowing for a few $\mathrm{dB}$ difference between results from different measurement campaigns. Without MRC, the required power is also comparable in case of space-time coding. However, beamforming without MRC requires $8.9 \mathrm{~dB}$ more power for the dual-array system, compared to a single-array. This could be attributed to interference between scattered signals from the front and back beam, resulting in a larger signal variance and hence a worse BER characteristic. Alamouti coding for the two beams can improve the performance here, combining the benefits of beamforming and space-time coding.

For the dual-array system in LoS conditions, beamforming without MRC requires $4.1 \mathrm{~dB}$ less transmit power than spacetime coding, because of the effective concentration of power in the direction of the receiver. However, for the single-array system, beamforming requires $8.9 \mathrm{~dB}$ more transmit power due to the shadowing by the human body, which is not eliminated with this configuration.

\section{CONCLUSION}

In LoS propagation conditions, the dual-array system is effective in eliminating the shadowing by the human body. This is confirmed by graphs displaying the evolution of the received $E_{b} / N_{0}$ for subsequent frames, for single- and dualarray systems.

Beamforming power gains of around $3 \mathrm{~dB}$, compared to space-time coding are realized in LoS as well as NLoS conditions, with or without receiver diversity. However, the variance of the received signal is often higher for beamforming than for space-time coding due to the total absence of diversity in the beamforming case. Without diversity at the transmitter, diversity should be implemented at the receiver to obtain a low bit error rate at the receiver, without drastically increasing the transmit power.

Focusing on the required transmit power to obtain a given bit error rate $\left(\mathrm{BER}=10^{-4}\right)$ at the MRC receiver, the beamforming versus space-time coding performance is comparable for dual- as well as single-array systems. Note, however, that beamforming can be implemented using simple power splitters whereas space-time coding requires separate transmitter chains and is hence a much more expensive solution. In NLoS the required transmit power is only slightly higher for the singlearray system, but for LoS conditions the dual-array system requires around $15 \mathrm{~dB}$ less power for an equal bit error rate at the MRC receiver. For off-body beamforming a dual-array front and back antenna system is recommended, combined with receiver diversity.

\section{REFERENCES}

[1] C. Hermosilla, R. Valenzuela, L. Ahumada, and R. Feick, "Empirical comparison of MIMO and beamforming schemes for outdoor-indoor scenarios," Wireless Communications, IEEE Transactions on, vol. 8, no. 3, pp. $1139-1143$, march 2009.

[2] M. Scarpello, D. Vande Ginste, H. Rogier, "Design of a low-cost steerable textile array operating in varying relative humidity conditions", Accepted in August 2011 for publication in Microwave and Optical Technology Letters, Wiley.

[3] P. Van Torre P., M. Scarpello, L. Vallozzi, H. Rogier, M. Moeneclaey, D. Vande Ginste, J. Verhaevert, "Indoor Off-Body Wireless Communication: Static Beamforming versus Space-Time Coding", Accepted in October 2011 for publication in the International Journal of Antennas and Propagation - Special Issue on MIMO Antenna Design and Channel Modeling. 